\title{
A bound on the second Betti number of hyperkähler manifolds of complex dimension six*
}

\author{
Justin Sawon \\ July, 2020
}

\begin{abstract}
Let $M$ be an irreducible compact hyperkähler manifold of complex dimension six. Under an assumption on the Looijenga-Lunts-Verbitsky decomposition of the cohomology of $M$, we prove that the second Betti number of $M$ is at most 23 .
\end{abstract}

\section{Introduction}

An irreducible hyperkähler manifold is a Riemannian manifold of real dimension $4 n$ whose holonomy is equal to $\operatorname{Sp}(n)$. The Riemannian metric will be Kählerian with respect to an $S^{2}$-family of complex structures, so henceforth we will always use the complex dimension, 2n. Beauville [2] and Guan [7] independently proved that the second Betti number of an irreducible compact hyperkähler manifold of dimension four is bounded above by 23. The Hilbert scheme of two points on a K3 surface has second Betti number 23, so this bound is sharp.

Looijenga and Lunts [1] and Verbitsky [17] showed that the cohomology of a hyperkähler manifold admits an action of $\mathfrak{s o}\left(4, b_{2}-2\right)$, where $b_{2}$ is the second Betti number. In this article we prove that the second Betti number of an irreducible compact hyperkähler manifold of dimension six is also bounded above by 23 , under the assumption that only certain irreducible $\mathfrak{s o}\left(4, b_{2}-2\right)$-representations appear in the Looijenga-Lunts-Verbitsky decomposition; see Theorem 3 for the precise statement. Up to deformation, there are currently three known examples of such manifolds: the Hilbert scheme of three points on a K3 surface, the generalized Kummer variety (see Beauville [1]), and an example of O'Grady [14]. These examples have second Betti numbers 23, 7, and 8, respectively, so once again our bound is sharp. Moreover, these examples all satisfy the assumption on the Looijenga-Lunts-Verbitsky decomposition of the cohomology.

Using the same ideas, we prove that the second Betti number of an irreducible compact hyperkähler manifold of dimension eight is bounded above by 24, once again under an assumption on the LooijengaLunts-Verbitsky decomposition and assuming that all odd Betti numbers vanish; see Theorem 4 for the precise statement. In this dimension, the only examples currently known are the Hilbert scheme of four points on a K3 surface and the generalized Kummer variety, which have second Betti numbers 23 and 7 , respectively. In particular, it is possible that no example exists in dimension eight with second Betti number 24.

Why is it important to bound the second Betti number? The first Pontryagin class $p_{1}(M)$ determines a homogeneous polynomial of degree $2 n-2$ on $\mathrm{H}^{2}(M, \mathbb{Z})$, given by $\alpha \mapsto \int_{M} \alpha^{2 n-2} p_{1}(M)$. Huybrechts $[8$ proved that if the second integral cohomology $\mathrm{H}^{2}$ and the homogeneous polynomial of degree $2 n-2$ on $\mathrm{H}^{2}$ determined by the first Pontryagin class are fixed, then up to diffeomorphism there are only finitely many irreducible compact hyperkähler manifolds of dimension $2 n$ realizing this structure. (Instead, one

\footnotetext{
*2010 Mathematics Subject Classification. 53C26.
} 
can fix $\mathrm{H}^{2}$ and a certain normalization $\tilde{q}$ of the Beauville-Bogomolov quadratic form on $\mathrm{H}^{2}$ and arrive at the same conclusion; see 8. 8 .) A universal bound on the second Betti number in dimension $2 n$ would mean that there are finitely many possibilities for $\mathrm{H}^{2}$ as a $\mathbb{Z}$-module; it would then remain to bound the other data on $\mathrm{H}^{2}$, to conclude that there are finitely many diffeomorphism types of irreducible compact hyperkähler manifolds of that dimension.

The author would like to thank Colleen Robles for pointing out an error in an earlier version of this article: we had overlooked some irreducible representations that could appear in the Looijenga-LuntsVerbitsky decomposition of the cohomology. The author would also like to thank Nikon Kurnosov for conversations on this work, and the NSF for support through grants DMS-1206309 and DMS-1555206.

\section{Dimension four}

Let us recall how to bound the second Betti number in dimension four. Salamon [15] proved that the Betti numbers of a compact hyperkähler manifold of dimension $2 n$ satisfy the relation

$$
2 \sum_{j=1}^{2 n}(-1)^{j}\left(3 j^{2}-n\right) b_{2 n-j}=n b_{2 n} .
$$

Theorem 1 (Beauville [2], Guan [7]) Let $M$ be an irreducible compact hyperkähler manifold of complex dimension four. Then the second Betti number $b_{2}$ of $M$ is at most 23.

Proof Irreducible hyperkähler manifolds are simply-connected, so $b_{1}=0$. Therefore Salamon's relation for $n=2$ gives

$$
-2 b_{3}+20 b_{2}+92=2 b_{4} .
$$

Verbitsky [16] proved that $\operatorname{Sym}^{k} \mathrm{H}^{2}(M, \mathbb{R})$ injects into $\mathrm{H}^{2 k}(M, \mathbb{R})$ for $k \leq n$. In particular, we can write

$$
\mathrm{H}^{4}(M, \mathbb{R}) \cong \operatorname{Sym}^{2} \mathrm{H}^{2}(M, \mathbb{R}) \oplus \mathrm{H}_{\text {prim }}^{4}(M, \mathbb{R})
$$

and

$$
b_{4}=\left(\begin{array}{c}
b_{2}+1 \\
2
\end{array}\right)+b_{4}^{\prime}
$$

where $b_{4}^{\prime}$ denotes the dimension of the primitive cohomology $\mathrm{H}_{\text {prim }}^{4}(M, \mathbb{R})$. Substituting this into Salamon's relation gives

$$
-2 b_{3}+20 b_{2}+92=b_{2}\left(b_{2}+1\right)+2 b_{4}^{\prime},
$$

and therefore

$$
-\left(b_{2}+4\right)\left(b_{2}-23\right)=-b_{2}^{2}+19 b_{2}+92=2 b_{4}^{\prime}+2 b_{3} .
$$

The left-hand side is negative if $b_{2}>23$, whereas the right-hand side is clearly non-negative. Therefore the second Betti number $b_{2}$ can be at most 23 .

Example Up to deformation, there are two known examples of irreducible compact hyperkähler manifolds of dimension four: the Hilbert scheme $\mathrm{Hilb}^{2} S$ of two points on a K3 surface $S$ (see Fujiki [3]) and the generalized Kummer variety $K_{2}(A)$ of an abelian surface $A$ (see Beauville 11). Their Hodge diamonds 
are

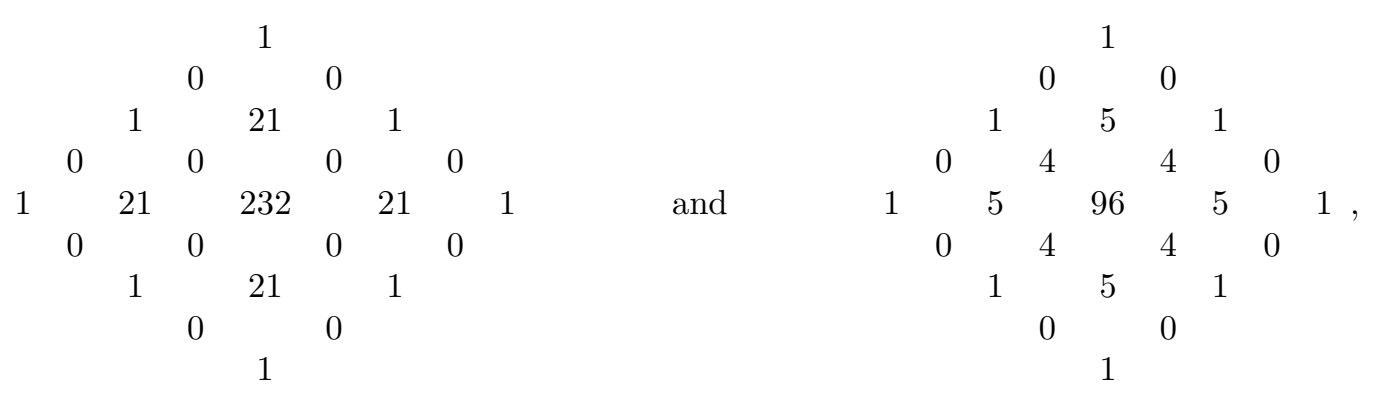

with $b_{2}=23, b_{3}=0, b_{4}^{\prime}=0$, and $b_{2}=7, b_{3}=8, b_{4}^{\prime}=80$, respectively. In fact, it follows from the proof above that if $b_{2}=23$ then $b_{3}$ and $b_{4}^{\prime}$ must both vanish.

\section{Dimension six}

In higher dimensions, the injection $\operatorname{Sym}^{k} \mathrm{H}^{2}(M, \mathbb{R}) \hookrightarrow \mathrm{H}^{2 k}(M, \mathbb{R})$ is insufficient to produce a bound on the second Betti number. Instead we employ the following refinement.

Theorem 2 (Looijenga and Lunts [11], Verbitsky [17]) Let $M$ be an irreducible compact hyperkähler manifold of dimension $2 n$ with second Betti number $b_{2}$. Then there is an action of $\mathfrak{s o}\left(4, b_{2}-2\right)$ on the real cohomology $\bigoplus_{k=0}^{4 n} \mathrm{H}^{k}(M, \mathbb{R})$, and hence of $\mathfrak{s o}\left(b_{2}+2, \mathbb{C}\right)$ on the complex cohomology $\bigoplus_{k=0}^{4 n} \mathrm{H}^{k}(M, \mathbb{C})$.

Remark This action is generated by Lefschetz operators: for each Kähler class $[\omega]$ the operators $L_{[\omega]}$ and $\Lambda_{[\omega]}$ generate an $\mathfrak{s l}(2, \mathbb{C})$-action on the complex cohomology, and the amalgamation of all these actions yields the $\mathfrak{s o}\left(b_{2}+2, \mathbb{C}\right)$-action.

We can decompose $\bigoplus_{k=0}^{4 n} \mathrm{H}^{k}(M, \mathbb{C})$ into irreducible representations for this $\mathfrak{s o}\left(b_{2}+2, \mathbb{C}\right)$-action. Their highest weights are related to Hodge bi-degrees; indeed, the Hodge diamond is the projection onto a plane of the (higher-dimensional) weight lattice of $\mathfrak{s o}\left(b_{2}+2, \mathbb{C}\right)$. We can choose positive roots so that the dominant Weyl chamber projects onto the shaded octant of the Hodge diamond shown in Figure 1 .

The irreducible representation with highest weight vector $1 \in \mathrm{H}^{0}(M, \mathbb{C})$ is precisely the subring of the cohomology generated by $\mathrm{H}^{2}(M, \mathbb{C})$. In dimension six, the remainder of the cohomology comes from irreducible representations whose highest weight vectors lie in the Hodge groups that are circled in Figure 1. By considering all irreducible representations of $\mathfrak{s o}\left(b_{2}+2, \mathbb{C}\right)$ (see Fulton and Harris [4), and observing how their highest weights project to the Hodge diamond, we conclude that the only irreducible representations that could appear are those described in Table 1. In the second column of this table the highest weights are given in terms of the fundamental weights. In the fourth column $\mathbb{C}^{b_{2}+2}$ and $\mathbb{C}$ denote the standard and trivial representations of $\mathfrak{s o}\left(b_{2}+2, \mathbb{C}\right)$, respectively. Thus $V_{k}$ is the representation given by the $k$ th exterior power $\Lambda^{k} \mathbb{C}^{b_{2}+2}$ of the standard representation of $\mathfrak{s o}\left(b_{2}+2, \mathbb{C}\right)$. Not shown in the table is that when $b_{2}+2=2 m+1$ is odd the largest exterior power $\Lambda^{m} \mathbb{C}^{b_{2}+2}$ has highest weight $2 \omega_{m}$. In addition, when $b_{2}+2=2 m$ is even the exterior power $\Lambda^{m-1} \mathbb{C}^{b_{2}+2}$ has highest weight $\omega_{m-1}+\omega_{m}$ while the middle degree exterior power $\Lambda^{m} \mathbb{C}^{b_{2}+2}$ is not irreducible; instead it decomposes into two irreducible representations of equal dimensions, $\Lambda^{m} \mathbb{C}^{b_{2}+2}=\Lambda_{+}^{m} \mathbb{C}^{b_{2}+2} \oplus \Lambda_{-}^{m} \mathbb{C}^{b_{2}+2}$, with highest weights $2 \omega_{m-1}$ and $2 \omega_{m}$, respectively. Note that tensor representations occur in even degrees in cohomology, while half-spin representations occur in odd degrees. The highest weights and dimensions of the latter are not needed for our arguments.

Example We can calculate the dimensions of the weight spaces of these representations. The highest weight vector of $V_{1}$ lies in $\mathrm{H}^{2,2}(M)$. Acting on this with Lefschetz operators $L_{[\omega]}$ gives us classes in 


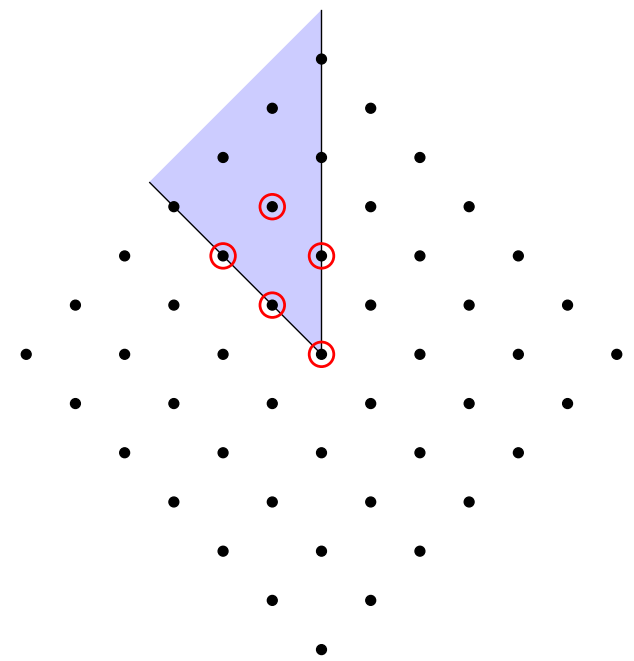

Figure 1: The Hodge diamond in dimension six

\begin{tabular}{|c|c|c|c|c|}
\hline & highest weight & highest weight vector in & $\mathfrak{s o}\left(b_{2}+2, \mathbb{C}\right)$-module & dimension \\
\hline$U_{\bullet}$ & & $\mathrm{H}^{2,1}(M)$ & half-spin representations & \\
$V_{1}$ & $\omega_{1}$ & $\mathrm{H}^{2,2}(M)$ & $\mathbb{C}^{b_{2}+2}$ & $b_{2}+2$ \\
$V_{2}$ & $\omega_{2}$ & $\mathrm{H}^{3,1}(M)$ & $\Lambda^{2} \mathbb{C}^{b_{2}+2}$ & $\left(^{b_{2}+2}\right.$ \\
$V_{3}$ & $\omega_{3}$ & $\mathrm{H}^{3,1}(M)$ & $\Lambda^{3} \mathbb{C}^{b_{2}+2}$ & $\left(^{2}+2\right.$ \\
$V_{4}$ & $\omega_{4}$ & $\mathrm{H}^{3,1}(M)$ & $\Lambda^{4} \mathbb{C}^{b_{2}+2}$ & $\left(\begin{array}{c}b_{2}+2 \\
4\end{array}\right)$ \\
$\vdots$ & $\vdots$ & $\vdots$ & $\vdots$ & $\vdots$ \\
$V_{k}$ & $\omega_{k}$ & $\mathrm{H}^{3,1}(M)$ & $\Lambda^{k} \mathbb{C}^{b_{2}+2}$ & $\left(\begin{array}{c}b_{2}+2 \\
k\end{array}\right)$ \\
$\vdots$ & $\vdots$ & $\vdots$ & $\vdots$ & $\vdots$ \\
$W \bullet$ & & $\mathrm{H}^{3,2}(M)$ & half-spin representations & \\
$T$ & 0 & $\mathrm{H}^{3,3}(M)$ & $\mathbb{C}$ & 1 \\
\hline
\end{tabular}

Table 1: Irreducible representations of $\mathfrak{s o}\left(b_{2}+2, \mathbb{C}\right)$ that could occur in the cohomology of $M$

$\mathrm{H}^{4,2}(M), \mathrm{H}^{3,3}(M), \mathrm{H}^{2,4}(M)$, and $\mathrm{H}^{4,4}(M)$, and indeed we find that $V_{1}$ will sit inside the Hodge diamond 
in the following manner (where we have indicated the dimension of $V_{1}^{p, q}$ for each $p, q$ )

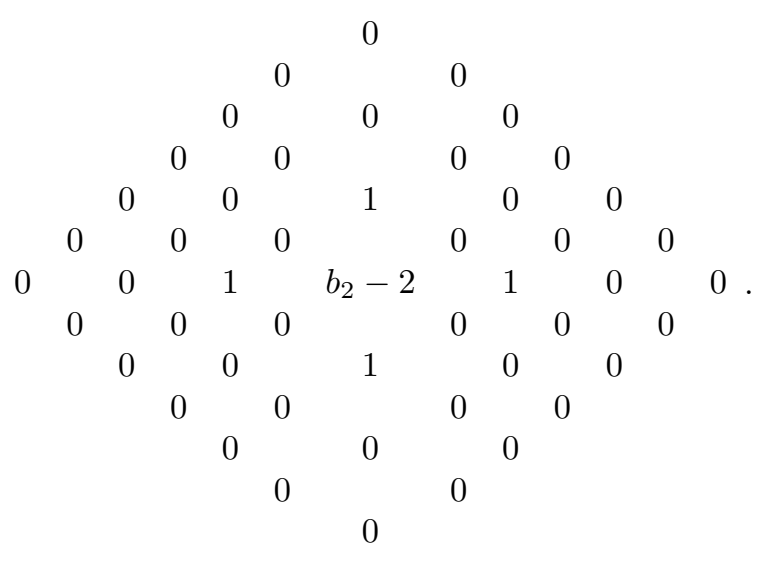

The weight decomposition of the exterior power $V_{k}=\Lambda^{k} V_{1}$ for $k \geq 2$ is derived from the weight decomposition of $V_{1}$. Because the Hodge bi-degrees are derived from the weights, we can thereby determine the dimensions of the $V_{k}^{p \cdot q}$ s. For example,

$$
\operatorname{dim} V_{2}^{2,2}=\operatorname{dim} V_{1}^{2,2} \operatorname{dim} V_{1}^{3,3} .
$$

(Note that weight zero corresponds to Hodge bi-degree $(3,3)$; if we shift by $(3,3)$ then the bi-degrees would become additive.) We find that $V_{2}$ will sit inside the Hodge diamond as

$$
\begin{aligned}
& 0
\end{aligned}
$$

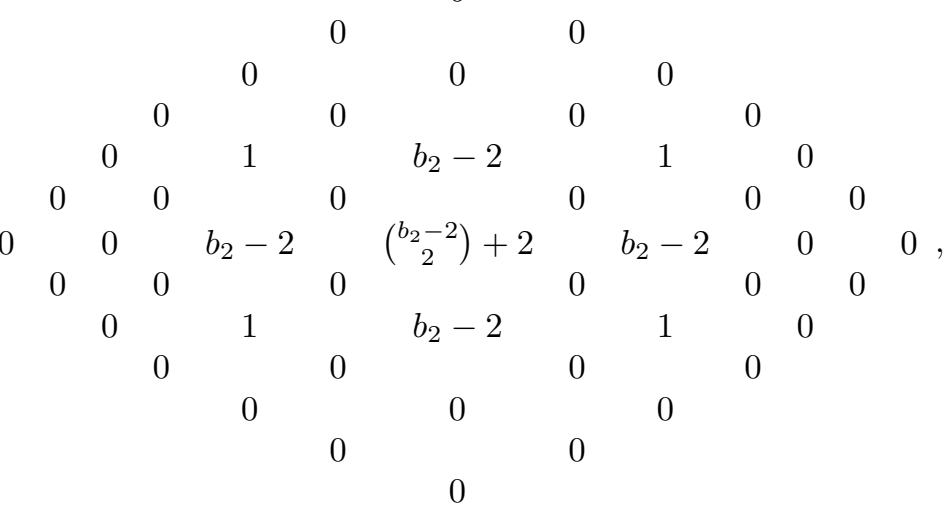

$V_{3}$ will sit inside the Hodge diamond as

$$
\begin{aligned}
& 0
\end{aligned}
$$

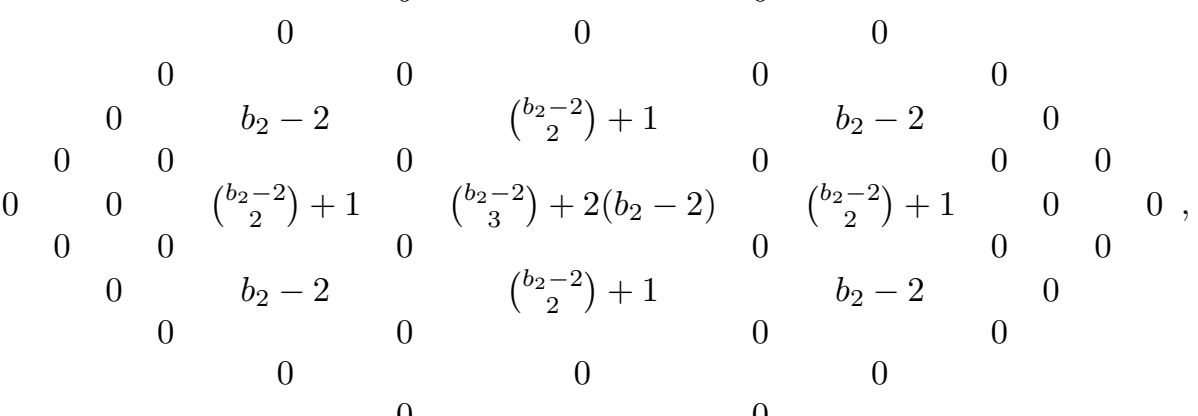

$$
\begin{aligned}
& 0 \\
& 0
\end{aligned}
$$


and $V_{4}$ will sit inside the Hodge diamond as

$$
\begin{aligned}
& 0 \\
& 0 \text { 0 } \\
& \begin{array}{ccccc} 
& & & & 0 \\
& & & 0 & \left(\begin{array}{c}
b_{2}-2 \\
2
\end{array}\right) \\
& & 0 & & \\
& 0 & & 0 & \\
0 & & 0 & & \left(\begin{array}{c}
b_{2}-2 \\
3
\end{array}\right)+b_{2}-2 \\
& 0 & & 0 & \\
& & 0 & & \left(\begin{array}{c}
b_{2}-2 \\
2
\end{array}\right) \\
& & & 0 &
\end{array} \\
& \left(\begin{array}{c}
b_{2}-2 \\
3
\end{array}\right)+b_{2}-2 \\
& \begin{array}{c}
\left(\begin{array}{c}
b_{2}-2 \\
4
\end{array}\right)+2\left(\begin{array}{c}
b_{2}-2 \\
2
\end{array}\right)+1 \\
\left(\begin{array}{c}
b_{2}-2 \\
3
\end{array}\right)+b_{2}-2
\end{array}
\end{aligned}
$$

Table 2 gives the dimensions of the intersections of these representations with $\mathrm{H}^{4}(M, \mathbb{C})$ and $\mathrm{H}^{6}(M, \mathbb{C})$.

\begin{tabular}{|c|c|c|c|}
\hline & dimension & dimension of $V_{\bullet} \cap \mathrm{H}^{4}(M, \mathbb{C})$ & dimension of $V_{\bullet} \cap \mathrm{H}^{6}(M, \mathbb{C})$ \\
\hline$V_{1}$ & $b_{2}+2$ & $\left(\begin{array}{c}b_{2} \\
0\end{array}\right)=1$ & $\left(\begin{array}{c}b_{2} \\
1\end{array}\right)=b_{2}$ \\
$V_{2}$ & $\left(\begin{array}{c}b_{2}+2 \\
2\end{array}\right)$ & $\left(\begin{array}{c}b_{2} \\
1\end{array}\right)=b_{2}$ & $\left(\begin{array}{c}b_{2} \\
2\end{array}\right)+\left(\begin{array}{c}b_{2} \\
0\end{array}\right)=\frac{b_{2}^{2}-b_{2}+2}{2}$ \\
$V_{3}$ & $\left(\begin{array}{c}b_{2}+2 \\
3\end{array}\right)$ & $\left(\begin{array}{c}b_{2} \\
2\end{array}\right)=\frac{b_{2}\left(b_{2}-1\right)}{2}$ & $\left(\begin{array}{c}b_{2} \\
3\end{array}\right)+\left(\begin{array}{c}b_{2} \\
1\end{array}\right)=\frac{b_{2}\left(b_{2}^{2}-3 b_{2}+8\right)}{6}$ \\
$V_{4}$ & $\left(\begin{array}{c}b_{2}+2 \\
4\end{array}\right)$ & $\left(\begin{array}{c}b_{2} \\
3\end{array}\right)=\frac{b_{2}\left(b_{2}-1\right)\left(b_{2}-2\right)}{6}$ & $\left(\begin{array}{c}b_{2} \\
4\end{array}\right)+\left(\begin{array}{c}b_{2} \\
2\end{array}\right)=\frac{b_{2}\left(b_{2}-1\right)\left(b_{2}^{2}-5 b_{2}+18\right)}{24}$ \\
$\vdots$ & $\vdots$ & $\vdots$ & $\vdots$ \\
$V_{k}$ & $\left(\begin{array}{c}b_{2}+2 \\
k\end{array}\right)$ & $\left(\begin{array}{c}b_{2} \\
k-1\end{array}\right)$ & $\left(\begin{array}{c}b_{2} \\
k\end{array}\right)+\left(\begin{array}{c}b_{2} \\
k-2\end{array}\right)$ \\
& & & \\
\hline
\end{tabular}

Table 2: Dimensions of $V_{\bullet}$ in degrees 4 and 6

With these preliminaries out of the way, we can prove our main result.

Theorem 3 Let $M$ be an irreducible compact hyperkähler manifold of complex dimension six. Of the possible irreducible representations of $\mathfrak{s o}\left(b_{2}+2, \mathbb{C}\right)$ with highest weight vectors in $\mathrm{H}^{2,2}(M)$ and $\mathrm{H}^{3,1}(M)$ in the Looijenga-Lunts-Verbitsky decomposition of the cohomology of $M$, assume that only $V_{1}, V_{2}$, and $V_{3}$ can appear (i.e., assume that $V_{4}, V_{5}, \ldots$ do not appear). Then the second Betti number $b_{2}$ of $M$ is at most 23.

Proof When $n=3$ Salamon's relation gives

$$
18 b_{4}-48 b_{3}+90 b_{2}+210=3 b_{6} .
$$

Decompose the complex cohomology of $M$ into irreducible representations of $\mathfrak{s o}\left(b_{2}+2, \mathbb{C}\right)$, as above. Suppose that $V_{1}$ occurs with multiplicity $c, V_{2}$ occurs with multiplicity $d, V_{3}$ occurs with multiplicity $e$, and the trivial representation $T=\mathbb{C}$ occurs with multiplicity $f$. The contributions of $V_{1}, V_{2}$, and $V_{3}$ to $\mathrm{H}^{4}(M, \mathbb{C})$ are of dimensions $1, b_{2}$, and $\frac{b_{2}\left(b_{2}-1\right)}{2}$, respectively. Including $\operatorname{Sym}^{2} \mathrm{H}^{2}(M, \mathbb{C})$ and multiplicities, we deduce that

$$
b_{4}=\left(\begin{array}{c}
b_{2}+1 \\
2
\end{array}\right)+c+d b_{2}+e\left(\frac{b_{2}\left(b_{2}-1\right)}{2}\right) .
$$


Similarly, the contributions of $V_{1}, V_{2}, V_{3}$, and $T=\mathbb{C}$ to $\mathrm{H}^{6}(M, \mathbb{C})$ are of dimensions $b_{2}, \frac{b_{2}^{2}-b_{2}+2}{2}$, $\frac{b_{2}\left(b_{2}^{2}-3 b_{2}+8\right)}{6}$, and 1 , respectively. Including $\operatorname{Sym}^{3} \mathrm{H}^{2}(M, \mathbb{C})$ and multiplicities, we deduce that

$$
b_{6}=\left(\begin{array}{c}
b_{2}+2 \\
3
\end{array}\right)+c b_{2}+d\left(\frac{b_{2}^{2}-b_{2}+2}{2}\right)+e\left(\frac{b_{2}\left(b_{2}^{2}-3 b_{2}+8\right)}{6}\right)+f .
$$

Substituting the formulae for $b_{4}$ and $b_{6}$ into Salamon's relation (and multiplying by 2 ) gives

$$
\begin{aligned}
36\left(\left(\begin{array}{c}
b_{2}+1 \\
2
\end{array}\right)+c+d b_{2}\right. & \left.+e\left(\frac{b_{2}\left(b_{2}-1\right)}{2}\right)\right)-96 b_{3}+180 b_{2}+420 \\
= & 6 b_{6}=6\left(\left(\begin{array}{c}
b_{2}+2 \\
3
\end{array}\right)+c b_{2}+d\left(\frac{b_{2}^{2}-b_{2}+2}{2}\right)+e\left(\frac{b_{2}\left(b_{2}^{2}-3 b_{2}+8\right)}{6}\right)+f\right),
\end{aligned}
$$

and after simplifying and rearranging we obtain

$$
\begin{aligned}
-\left(b_{2}+6\right)\left(b_{2}-\frac{21+\sqrt{721}}{2}\right) & \left(b_{2}-\frac{21-\sqrt{721}}{2}\right)=-b_{2}^{3}+15 b_{2}^{2}+196 b_{2}+420 \\
& =6 c\left(b_{2}-6\right)+3 d\left(b_{2}^{2}-13 b_{2}+2\right)+e b_{2}\left(b_{2}^{2}-21 b_{2}+26\right)+6 f+96 b_{3} .
\end{aligned}
$$

The left-hand side is negative if $b_{2} \geq 24>\frac{21+\sqrt{721}}{2} \approx 23.9257$. On the other hand, $c, d, e, f$, and $b_{3}$ are all non-negative, so the right-hand side will be non-negative for $b_{2} \geq 24$ (indeed, for $b_{2} \geq 20$ ). Therefore the second Betti number $b_{2}$ can be at most 23 .

Remark The contributions of $V_{4}$ to $\mathrm{H}^{4}(M, \mathbb{C})$ and $\mathrm{H}^{6}(M, \mathbb{C})$ have dimensions

$$
\frac{b_{2}\left(b_{2}-1\right)\left(b_{2}-2\right)}{6} \quad \text { and } \quad \frac{b_{2}\left(b_{2}-1\right)\left(b_{2}^{2}-5 b_{2}+18\right)}{24}
$$

respectively. For each occurrence of $V_{4}$ in the decomposition of the cohomology of $M$ we would need to add an additional term

$$
6 \frac{b_{2}\left(b_{2}-1\right)\left(b_{2}^{2}-5 b_{2}+18\right)}{24}-36 \frac{b_{2}\left(b_{2}-1\right)\left(b_{2}-2\right)}{6}=\frac{b_{2}\left(b_{2}-1\right)\left(b_{2}^{2}-29 b_{2}+66\right)}{4}
$$

to the right-hand side of the last displayed equation of the proof. If $b_{2}=24,25$, or 26 then this term would be negative and the proof would break down. However, we could still conclude that $b_{2} \geq 27$ is impossible.

More generally, the contributions of $V_{k}$ to $\mathrm{H}^{4}(M, \mathbb{C})$ and $\mathrm{H}^{6}(M, \mathbb{C})$ have dimensions

$$
\left(\begin{array}{c}
b_{2} \\
k-1
\end{array}\right) \quad \text { and } \quad\left(\begin{array}{c}
b_{2} \\
k
\end{array}\right)+\left(\begin{array}{c}
b_{2} \\
k-2
\end{array}\right)
$$

respectively. For each occurrence of $V_{k}$ in the decomposition of the cohomology of $M$ we would need to add an additional term

$$
6\left(\begin{array}{c}
b_{2} \\
k
\end{array}\right)+6\left(\begin{array}{c}
b_{2} \\
k-2
\end{array}\right)-36\left(\begin{array}{c}
b_{2} \\
k-1
\end{array}\right)=\frac{6 b_{2}\left(b_{2}-1\right) \cdots\left(b_{2}-k+3\right)}{k !}\left(b_{2}^{2}-(8 k-3) b_{2}+\left(8 k^{2}-16 k+2\right)\right)
$$

to the right-hand side of the last displayed equation of the proof. Calculating the roots of the quadratic factor, we see that if

$$
b_{2} \geq \frac{8 k-3+\sqrt{32 k^{2}+16 k+1}}{2}
$$

then this additional term will be non-negative, and we again reach the desired contradiction. Thus allowing $V_{1}, V_{2}, \ldots, V_{k}$ to appear in the Looijenga-Lunts-Verbitsky decomposition of the cohomology of $M$, for some $k \geq 4$, we still obtain an upper bound on $b_{2}$, but unfortunately this bound grows roughly linearly with $k$. 
Example Up to deformation, there are three known examples of irreducible compact hyperkähler manifolds of dimension six: the Hilbert scheme $\mathrm{Hilb}^{3} S$ of three points on a K3 surface $S$, the generalized Kummer variety $K_{3}(A)$ of an abelian surface $A$ (see Beauville [1]), and an example $M_{6}$ of O'Grady [14. The Hodge numbers of Hilbert schemes of points on K3 surfaces and of generalized Kummer varieties were calculated by Göttsche and Soergel [5]; for $\operatorname{Hilb}^{3} S$ and $K_{3}(A)$ they are

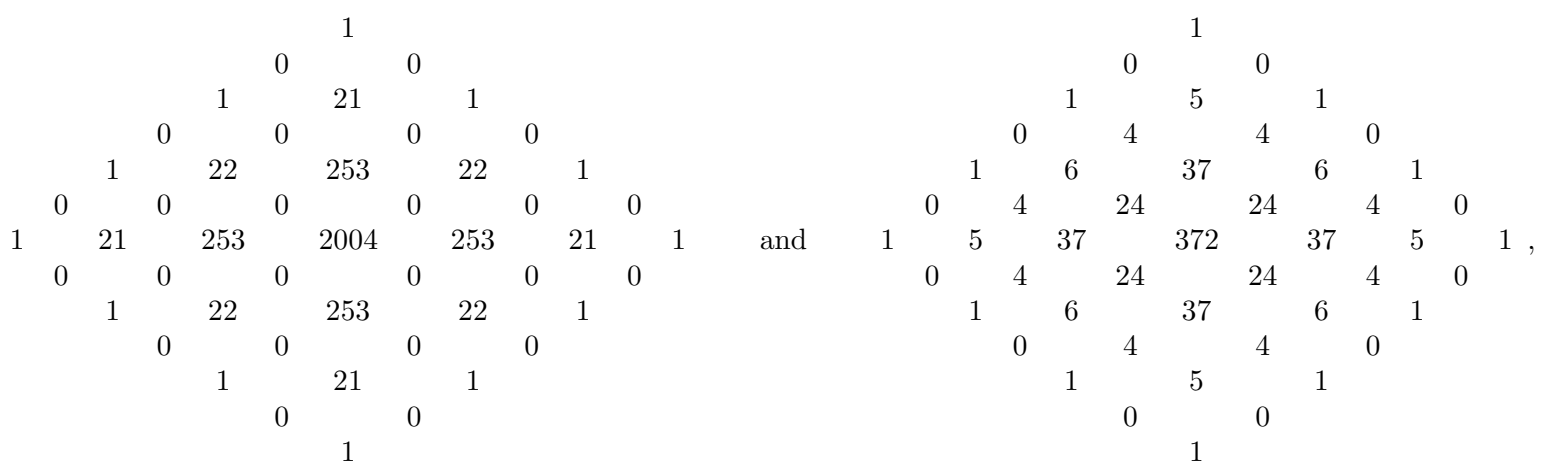

with $b_{2}=23, b_{3}=0, d=1, c=e=f=0$, and $b_{2}=7, b_{3}=8, c=16, d=1, e=0, f=240$, respectively. In particular, $V_{4}, V_{5}, \ldots$ do not appear in the Looijenga-Lunts-Verbitsky decomposition of the cohomology of $\mathrm{Hilb}^{3} S$ and $K_{3}(A)$. Indeed, $e=0$ for both, so $V_{3}$ also does not appear.

The Hodge numbers of O'Grady's example $M_{6}$ were calculated by Mongardi, Rapagnetta, and Saccà [12] they are

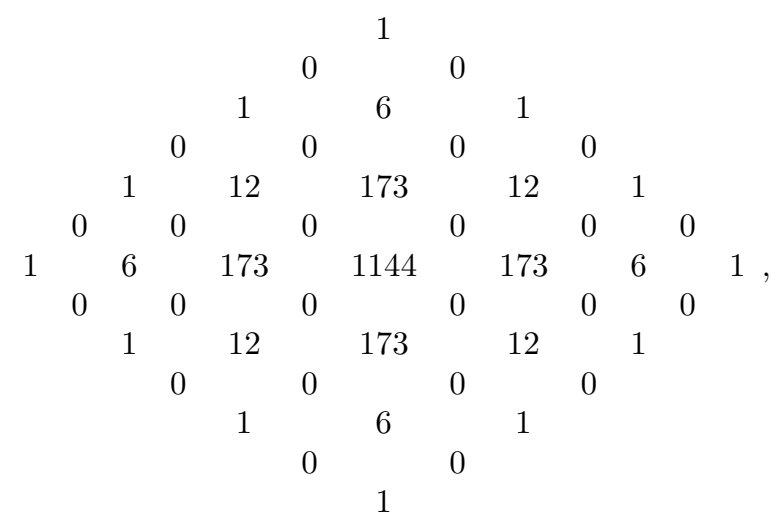

with $b_{2}=8, b_{3}=0$. A priori there are two different Looijenga-Lunts-Verbitsky decompositions into irreducible $\mathfrak{s o}(10, \mathbb{C})$-representations that could produce this Hodge diamond: either $c=115, d=6$, $e=0, f=290$ or $c=135, d=0, e=1, f=240$. (Note that in neither case do $V_{4}, V_{5}, \ldots$ appear.) In fact, Green, Kim, Laza, and Robles [6] have determined that the latter is the correct decomposition, but this is not immediate from the representation theory and it requires geometric arguments.

Remark The hypothesis of Theorem 3 that $V_{4}, V_{5}, \ldots$ do not appear in the decomposition of the cohomology of $M$ was originally introduced somewhat artificially, to make the proof work. Indeed the remark above shows that allowing $V_{4}, V_{5}, \ldots$ to appear leads to progressively weaker bounds on $b_{2}$. Nevertheless, the hypothesis is satisfied for all known examples in dimension six, as observed above.

A more conceptual justification of the hypothesis is provided by Green, Kim, Laza, and Robles [6], by relating it to a conjecture of Nagai [13] concerning monodromy operators for one-parameter degenerations of hyperkähler manifolds. Green, et al. show that Nagai's conjecture is equivalent to a certain restriction on the highest weight vectors in the Looijenga-Lunts-Verbitsky decomposition of the cohomology in even degrees, and they verify that this restriction (and hence Nagai's conjecture) holds for all known 
hyperkähler manifolds, in all dimensions. They then recognize that there is a stronger restriction on the highest weight vectors that is more natural, which also holds for all known hyperkähler manifolds. This (conjectural) stronger restriction reduces to our hypothesis in dimension six. See [6] for details.

\section{$4 \quad$ Higher dimensions}

When $n=4$ Salamon's relation gives

$$
2 b_{7}+16 b_{6}-46 b_{5}+88 b_{4}-142 b_{3}+208 b_{2}+376=4 b_{8} .
$$

Thus in dimension eight, $b_{7}$ appears with a coefficient of the 'wrong' sign, and we cannot simply imitate the proof of Theorem 3 . To proceed, we will assume that $b_{7}=0$. In fact, this is equivalent to assuming that all odd Betti numbers vanish, as the presence of cohomology in any odd degree will force $\mathrm{H}^{7}(M, \mathbb{C})$ to be non-vanishing because of the $\mathfrak{s o}\left(b_{2}+2, \mathbb{C}\right)$-action.

The Hodge diamond omitting the odd cohomology is shown in Figure 2, After removing the cohomology generated by $\mathrm{H}^{2}(M, \mathbb{C})$, we are left with irreducible representations whose highest weight vectors lie in the circled Hodge groups. The only irreducible representations that could appear are those described in Table 3. Like in dimension six, $V_{k}$ denotes that $k$ th exterior power $\Lambda^{k} \mathbb{C}^{b_{2}+2}$ of the standard representation, while $U_{1}$ is given by taking the 2 nd symmetric power $\mathrm{Sym}^{2} \mathbb{C}^{b_{2}+2}$ of the standard representation and removing the trivial direct summand $\mathbb{C}$, and $U_{k}$ is given by taking the tensor product $\mathbb{C}^{b_{2}+2} \otimes \Lambda^{k} \mathbb{C}^{b_{2}+2}$ of the standard representation with its $k$ th exterior power and removing the direct summands $\Lambda^{k+1} \mathbb{C}^{b_{2}+2}$ and $\Lambda^{k-1} \mathbb{C}^{b_{2}+2}$ (this leaves an irreducible representation with highest weight $\omega_{1}+\omega_{k}$, except in a few special cases described in the following remark).

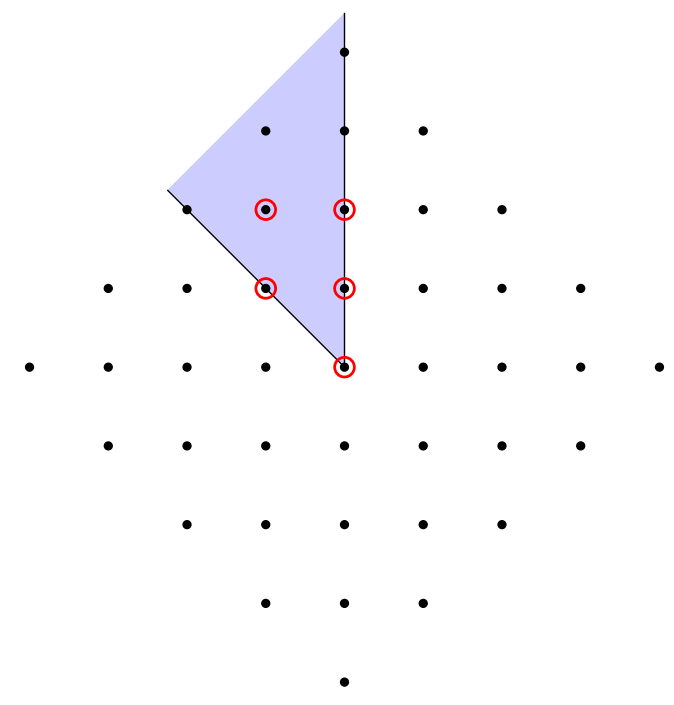

Figure 2: The Hodge diamond in dimension eight

Remark In Theorem 4 we will only allow irreducible representations $U_{k}$ and $V_{k}$ with $k$ small relative to $b_{2}$. Nevertheless, for completeness let us clarify that if $b_{2}+2=2 m+1$ is odd then $V_{m}=\Lambda^{m} \mathbb{C}^{b_{2}+2}$ has highest weight $2 \omega_{m}$ and $U_{m}$ has highest weight $\omega_{1}+2 \omega_{m}$. If $b_{2}+2=2 m$ is even then $V_{m-1}=\Lambda^{m-1} \mathbb{C}^{b_{2}+2}$ has highest weight $\omega_{m-1}+\omega_{m}, U_{m-1}$ has highest weight $\omega_{1}+\omega_{m-1}+\omega_{m}, \Lambda^{m} \mathbb{C}^{b_{2}+2}=\Lambda_{+}^{m} \mathbb{C}^{b_{2}+2} \oplus \Lambda_{-}^{m} \mathbb{C}^{b_{2}+2}$ decomposes into two irreducible representations of equal dimensions with highest weights $2 \omega_{m-1}$ and $2 \omega_{m}$, 


\begin{tabular}{|c|c|c|c|c|}
\hline & highest weight & highest weight vector in & $\mathfrak{s o}\left(b_{2}+2, \mathbb{C}\right)$-module & dimension \\
\hline$U_{1}$ & $2 \omega_{1}$ & $\mathrm{H}^{2,2}(M)$ & $\mathrm{Sym}^{2} \mathbb{C}^{b_{2}+2}-\mathbb{C}$ & $\frac{\left(\frac{\left(b_{2}+4\right)\left(b_{2}+1\right)}{2}\right.}{\left(b_{2}+4\right)\left(b_{2}+2\right) b_{2}}$ \\
$U_{2}$ & $\omega_{1}+\omega_{2}$ & $\mathrm{H}^{3,1}(M)$ & $\mathbb{C}^{b_{2}+2} \otimes \Lambda^{2} \mathbb{C}^{b_{2}+2}-\Lambda^{3} \mathbb{C}^{b_{2}+2}-\mathbb{C}^{b_{2}+2}$ & $\frac{\left(b_{2}+4\right)\left(b_{2}+2\right)\left(b_{2}+1\right)\left(b_{2}-1\right)}{8}$ \\
$U_{3}$ & $\omega_{1}+\omega_{3}$ & $\mathrm{H}^{3,1}(M)$ & $\mathbb{C}^{b_{2}+2} \otimes \Lambda^{3} \mathbb{C}^{b_{2}+2}-\Lambda^{4} \mathbb{C}^{b_{2}+2}-\Lambda^{2} \mathbb{C}^{b_{2}+2}$ & $\vdots$ \\
$\vdots$ & $\vdots$ & $\vdots$ & $\vdots$ & $\vdots$ \\
$U_{k}$ & $\omega_{1}+\omega_{k}$ & $\mathrm{H}^{3,1}(M)$ & $\mathbb{C}^{b_{2}+2} \otimes \Lambda^{k} \mathbb{C}^{b_{2}+2}-\Lambda^{k+1} \mathbb{C}^{b_{2}+2}-\Lambda^{k-1} \mathbb{C}^{b_{2}+2}$ & $\left(b_{2}+2\right)\left(\begin{array}{c}b_{2}+2 \\
k\end{array}\right)-\left(\begin{array}{c}b_{2}+2 \\
k+1\end{array}\right)-\left(\begin{array}{c}b_{2}+2 \\
k-1\end{array}\right)$ \\
$\vdots$ & $\vdots$ & $\vdots$ & $\vdots$ & $\vdots$ \\
$V_{1}$ & $\omega_{1}$ & $\mathrm{H}^{3,3}(M)$ & $\mathbb{C}^{b_{2}+2}$ & $b_{2}+2$ \\
$V_{2}$ & $\omega_{2}$ & $\mathrm{H}^{4,2}(M)$ & $\Lambda^{2} \mathbb{C}^{b_{2}+2}$ & $\left(\begin{array}{c}b_{2}+2 \\
2_{2}\end{array}\right)$ \\
$V_{3}$ & $\omega_{3}$ & $\mathrm{H}^{4,2}(M)$ & $\Lambda^{3} \mathbb{C}^{b_{2}+2}$ & $\left(\begin{array}{c}3 \\
b_{2}+2\end{array}\right)$ \\
$V_{4}$ & $\omega_{4}$ & $\mathrm{H}^{4,2}(M)$ & $\Lambda^{4} \mathbb{C}^{b_{2}+2}$ & $\vdots$ \\
$\vdots$ & $\vdots$ & $\vdots$ & $\vdots$ & $\left(\begin{array}{c}b_{2}+2 \\
k\end{array}\right)$ \\
$V_{k}$ & $\omega_{k}$ & $\mathrm{H}^{4,2}(M)$ & $\Lambda^{k} \mathbb{C}^{b_{2}+2}$ & $\vdots$ \\
$\vdots$ & $\vdots$ & $\vdots$ & $\vdots$ & 1 \\
\hline
\end{tabular}

Table 3: Irreducible representations of $\mathfrak{s o}\left(b_{2}+2, \mathbb{C}\right)$ that that could occur in the cohomology of $M$

and $\mathbb{C}^{b_{2}+2} \otimes \Lambda^{m} \mathbb{C}^{b_{2}+2}-\Lambda^{m+1} \mathbb{C}^{b_{2}+2}-\Lambda^{m-1} \mathbb{C}^{b_{2}+2}$ decomposes into two irreducible representations of equal dimensions with highest weights $\omega_{1}+2 \omega_{m-1}$ and $\omega_{1}+2 \omega_{m}$.

Table 4 gives the dimensions of the intersections of these representations with $\mathrm{H}^{4}(M, \mathbb{C}), \mathrm{H}^{6}(M, \mathbb{C})$, and $\mathrm{H}^{8}(M, \mathbb{C})$. For instance, to compute these dimensions for $U_{k}$ we use the description

$$
U_{k}=\mathbb{C}^{b_{2}+2} \otimes \Lambda^{k} \mathbb{C}^{b_{2}+2}-\Lambda^{k+1} \mathbb{C}^{b_{2}+2}-\Lambda^{k-1} \mathbb{C}^{b_{2}+2}
$$

with $\mathbb{C}^{b_{2}+2}=\mathbb{C} \oplus \mathbb{C}^{b_{2}} \oplus \mathbb{C}$ graded by $-2,0$, and 2. After an overall shift of 8 , this induces the required grading on $U_{k}$.

\begin{tabular}{|c|c|c|c|}
\hline & dimension of $\cap \mathrm{H}^{4}(M, \mathbb{C})$ & dimension of $\cap \mathrm{H}^{6}(M, \mathbb{C})$ & dimension of $\cap \mathrm{H}^{8}(M, \mathbb{C})$ \\
\hline$U_{1}$ & $\left(\begin{array}{c}b_{2} \\
0\end{array}\right)=1$ & $b_{2}\left(\begin{array}{c}b_{2} \\
0\end{array}\right)=b_{2}$ & $b_{2}\left(\begin{array}{c}b_{2} \\
1\end{array}\right)-\left(\begin{array}{c}b_{2} \\
2\end{array}\right)=\frac{\left(b_{2}+1\right) b_{2}}{2}$ \\
$U_{2}$ & $\left(\begin{array}{c}b_{2} \\
1\end{array}\right)=b_{2}$ & $b_{2}\left(\begin{array}{c}b_{2} \\
1\end{array}\right)=b_{2}^{2}$ & $b_{2}\left(\begin{array}{c}b_{2} \\
2\end{array}\right)+b_{2}\left(\begin{array}{c}b_{2} \\
0\end{array}\right)-\left(\begin{array}{c}b_{2} \\
3\end{array}\right)=\frac{b_{2}\left(b_{2}^{2}+2\right)}{3}$ \\
$U_{3}$ & $\left(\begin{array}{c}b_{2} \\
2\end{array}\right)=\frac{b_{2}\left(b_{2}-1\right)}{2}$ & $b_{2}\left(\begin{array}{c}b_{2} \\
2\end{array}\right)=\frac{b_{2}^{2}\left(b_{2}-1\right)}{2}$ & $b_{2}\left(\begin{array}{c}b_{2} \\
3\end{array}\right)+b_{2}\left(\begin{array}{c}b_{2} \\
1\end{array}\right)-\left(\begin{array}{c}b_{2} \\
4\end{array}\right)-\left(\begin{array}{c}b_{2} \\
0\end{array}\right)=\frac{\left(b_{2}+1\right)\left(b_{2}-1\right)\left(b_{2}^{2}-2 b_{2}+8\right)}{8}$ \\
$\vdots$ & $\vdots$ & $\vdots$ & $\vdots$ \\
$U_{k}$ & $\left(\begin{array}{c}b_{2} \\
k-1\end{array}\right)$ & $b_{2}\left(\begin{array}{c}b_{2} \\
k-1\end{array}\right)$ & $b_{2}\left(\begin{array}{c}b_{2} \\
k\end{array}\right)+b_{2}\left(\begin{array}{c}b_{2} \\
k-2\end{array}\right)-\left(\begin{array}{c}b_{2} \\
k+1\end{array}\right)-\left(\begin{array}{c}b_{2} \\
k-3\end{array}\right)$ \\
$V_{1}$ & & $\left(\begin{array}{c}b_{2} \\
0\end{array}\right)=1$ & $\left(\begin{array}{c}b_{2} \\
1\end{array}\right)=b_{2}$ \\
$V_{2}$ & & $\left(\begin{array}{c}b_{2} \\
1\end{array}\right)=b_{2}$ & $\left(\begin{array}{c}b_{2} \\
2\end{array}\right)=\frac{b_{2}\left(b_{2}-1\right)}{2}$ \\
$V_{3}$ & & $\left(\begin{array}{c}b_{2} \\
3\end{array}\right)=\frac{b_{2}\left(b_{2}-1\right)\left(b_{2}-2\right)}{6}$ & $\left(\begin{array}{c}b_{2} \\
2\end{array}\right)+\left(\begin{array}{c}b_{2} \\
0\end{array}\right)=\frac{b_{2}^{2}-b_{2}+2}{2}$ \\
$V_{4}$ & & $\vdots$ & $\left(\begin{array}{c}b_{2} \\
3\end{array}\right)+\left(\begin{array}{c}b_{2} \\
1\end{array}\right)=\frac{b_{2}\left(b_{2}^{2}-3 b_{2}+8\right)}{3 b_{2}}$ \\
$\vdots$ & & $\left(\begin{array}{c}b_{2}-b_{2} \\
4\end{array}\right)+\left(\begin{array}{c}b_{2} \\
2\end{array}\right)=\frac{b_{2}\left(b_{2}-1\right)\left(b_{2}^{2}-5 b_{2}+18\right)}{24}$ \\
$V_{k}$ & & $\left(\begin{array}{c}b_{2} \\
k-1\end{array}\right)$ & $\vdots$ \\
& & & $\left(\begin{array}{c}b_{2} \\
k\end{array}\right)+\left(\begin{array}{c}b_{2} \\
k-2\end{array}\right)$ \\
\hline
\end{tabular}

Table 4: Dimensions of $U_{\bullet}$ and $V_{\bullet}$ in degrees 4,6 , and 8

Example The representation $U_{1}$ is generated by a highest weight vector in

$$
U_{1} \cap \mathrm{H}^{4}(M, \mathbb{C})=U_{1} \cap \mathrm{H}^{2,2}(M) \cong \mathbb{C},
$$


whereas

$$
U_{2} \cap \mathrm{H}^{4}(M, \mathbb{C}) \cong \mathbb{C}^{b_{2}}
$$

with

$$
\operatorname{dim}\left(U_{2} \cap \mathrm{H}^{3,1}(M)\right)=1=\operatorname{dim}\left(U_{2} \cap \mathrm{H}^{1,3}(M)\right) \quad \text { and } \quad \operatorname{dim}\left(U_{2} \cap \mathrm{H}^{2,2}(M)\right)=b_{2}-2 .
$$

Similarly

$$
U_{3} \cap \mathrm{H}^{4}(M, \mathbb{C}) \cong \Lambda^{2} \mathbb{C}^{b_{2}}
$$

with

$$
\operatorname{dim}\left(U_{3} \cap \mathrm{H}^{3,1}(M)\right)=b_{2}-2=\operatorname{dim}\left(U_{3} \cap \mathrm{H}^{1,3}(M)\right) \quad \text { and } \quad \operatorname{dim}\left(U_{3} \cap \mathrm{H}^{2,2}(M)\right)=\left(\begin{array}{c}
b_{2}-2 \\
2
\end{array}\right)+1 .
$$

In general

$$
U_{k} \cap \mathrm{H}^{4}(M, \mathbb{C}) \cong \Lambda^{k-1} \mathbb{C}^{b_{2}}
$$

with

$\operatorname{dim}\left(U_{k} \cap \mathrm{H}^{3,1}(M)\right)=\left(\begin{array}{c}b_{2}-2 \\ k-2\end{array}\right)=\operatorname{dim}\left(U_{k} \cap \mathrm{H}^{1,3}(M)\right)$ and $\operatorname{dim}\left(U_{k} \cap \mathrm{H}^{2,2}(M)\right)=\left(\begin{array}{c}b_{2}-2 \\ k-1\end{array}\right)+\left(\begin{array}{c}b_{2}-2 \\ k-3\end{array}\right)$.

We can now prove our result in dimension eight.

Theorem 4 Let $M$ be an irreducible compact hyperkähler manifold of complex dimension eight whose odd Betti numbers all vanish. Consider the part of the cohomology of $M$ not generated by $\mathrm{H}^{2}(M, \mathbb{C})$. Of the possible irreducible representations of $\mathfrak{s o}\left(b_{2}+2, \mathbb{C}\right)$ in its Looijenga-Lunts-Verbitsky decomposition, assume that only $U_{1}, U_{2}, U_{3}, V_{1}, V_{2}, V_{3}, V_{4}, V_{5}$, and $T$ can appear. Then the second Betti number $b_{2}$ of $M$ is at most 24 .

Proof The proof follows the same ideas as that of Theorem 3. When $n=4$ and all odd Betti numbers vanish, Salamon's relation gives

$$
16 b_{6}+88 b_{4}+208 b_{2}+376=4 b_{8} .
$$

The part of the complex cohomology of $M$ generated by $\mathrm{H}^{2}(M, \mathbb{C})$ contributes $\left(\begin{array}{c}b_{2}+1 \\ 2\end{array}\right),\left(\begin{array}{c}b_{2}+2 \\ 3\end{array}\right)$, and $\left(\begin{array}{c}b_{2}+3 \\ 4\end{array}\right)$ to $b_{4}, b_{6}$, and $b_{8}$, respectively. Writing the remainders of these Betti numbers as $b_{4}^{\prime}, b_{6}^{\prime}$, and $b_{8}^{\prime}$, Salamon's relation becomes

$$
\begin{aligned}
-4\left(\begin{array}{c}
b_{2}+3 \\
4
\end{array}\right)+16\left(\begin{array}{c}
b_{2}+2 \\
3
\end{array}\right)+88\left(\begin{array}{c}
b_{2}+1 \\
2
\end{array}\right)+208 b_{2}+376 & =4 b_{8}^{\prime}-16 b_{6}^{\prime}-88 b_{4}^{\prime} \\
& =4\left(b_{8}^{\prime}-4 b_{6}^{\prime}-22 b_{4}^{\prime}\right) .
\end{aligned}
$$

After simplifying and factoring, the left-hand side becomes

$$
-\frac{1}{6}\left(b_{2}+3\right)\left(b_{2}+8\right)\left(b_{2}-\frac{21+\sqrt{817}}{2}\right)\left(b_{2}-\frac{21-\sqrt{817}}{2}\right),
$$

which is negative if $b_{2} \geq 25>\frac{21+\sqrt{817}}{2} \approx 24.7916$. It remains to show that the right-hand is non-negative for $b_{2} \geq 25$.

First consider the contribution of $V_{k}$ to the right-hand side. Each occurrence of $V_{k}$ in the LooijengaLunts-Verbitsky decomposition contributes

$$
\left(\begin{array}{c}
b_{2} \\
k
\end{array}\right)+\left(\begin{array}{c}
b_{2} \\
k-2
\end{array}\right)-4\left(\begin{array}{c}
b_{2} \\
k-1
\end{array}\right)=\frac{b_{2}\left(b_{2}-1\right) \cdots\left(b_{2}-k+3\right)}{k !}\left(b_{2}^{2}-(6 k-3) b_{2}+\left(6 k^{2}-12 k+2\right)\right)
$$


to $b_{8}^{\prime}-4 b_{6}^{\prime}-22 b_{4}^{\prime}$. This contribution will be non-negative if

$$
b_{2} \geq \frac{6 k-3+\sqrt{12 k^{2}+12 k+1}}{2} .
$$

In particular, if $k=5$ we require $b_{2} \geq 23$, whereas if $k=6$ we would require $b_{2} \geq 28>\frac{33+\sqrt{505}}{2} \approx 27.7361$.

Next consider the contribution of $U_{k}$ to the right-hand side. Each occurrence of $U_{k}$ in the LooijengaLunts-Verbitsky decomposition contributes

$$
\begin{array}{r}
b_{2}\left(\begin{array}{c}
b_{2} \\
k
\end{array}\right)+b_{2}\left(\begin{array}{c}
b_{2} \\
k-2
\end{array}\right)-\left(\begin{array}{c}
b_{2} \\
k+1
\end{array}\right)-\left(\begin{array}{c}
b_{2} \\
k-3
\end{array}\right)-4 b_{2}\left(\begin{array}{c}
b_{2} \\
k-1
\end{array}\right)-22\left(\begin{array}{c}
b_{2} \\
k-1
\end{array}\right) \\
=\frac{b_{2}\left(b_{2}-1\right) \cdots\left(b_{2}-k+4\right)\left(b_{2}-k+2\right)\left(b_{2}+4\right)}{(k+1)(k-1) !}\left(b_{2}^{2}-(6 k+3) b_{2}+\left(6 k^{2}-12 k-16\right)\right)
\end{array}
$$

to $b_{8}^{\prime}-4 b_{6}^{\prime}-22 b_{4}^{\prime}$. This contribution will be non-negative if

$$
b_{2} \geq \frac{6 k+3+\sqrt{12 k^{2}+84 k+73}}{2} .
$$

In particular, if $k=3$ we require $b_{2} \geq 21>\frac{21+\sqrt{433}}{2} \approx 20.9043$, whereas if $k=4$ we would require $b_{2} \geq 26>\frac{27+\sqrt{601}}{2} \approx 25.7577$.

Finally, the contribution of the trivial representation $T$ to $b_{8}^{\prime}-4 b_{6}^{\prime}-22 b_{4}^{\prime}$ is just 1 , so always positive.

In conclusion, if we allow the irreducible representations $U_{1}, U_{2}, U_{3}, V_{1}, V_{2}, V_{3}, V_{4}, V_{5}$, and $T$ to appear in the Looijenga-Lunts-Verbitsky decomposition of the complex cohomology of $M$, then for $b_{2} \geq 25$ the right-hand side $4\left(b_{8}^{\prime}-4 b_{6}^{\prime}-22 b_{4}^{\prime}\right)$ of Salamon's relation will be non-negative, whereas the left-hand side will be negative. This contradiction proves that the second Betti number $b_{2}$ can be at most 24 .

Remark As the proof shows, if we allow $U_{4}$ to appear then we can conclude that $b_{2}$ can be at most 25 , and if we allow $V_{6}$ to appear then we can conclude that $b_{2}$ can be at most 27. Allowing $U_{k}$ and $V_{k}$ to appear for larger $k$, we still obtain upper bounds on $b_{2}$, but these bounds grows roughly linearly with $k$.

Remark Following the same steps in higher dimensions, the pattern appears to be that in dimension $2 n$, the polynomial in $b_{2}$ on the left-hand side has largest root $\frac{21+\sqrt{433+96 n}}{2}$. Given an irreducible compact hyperkähler manifold whose odd Betti numbers all vanish, we expect one can show that its second Betti number must satisfy $b_{2} \leq \frac{21+\sqrt{433+96 n}}{2}$, under some restrictions on the irreducible representations appearing in the Looijenga-Lunts-Verbitsky decomposition of its cohomology. The author has not rigorously verified this, though this direction has been pursued by Kurnosov [10, and indeed, Kim and Laza [9] later identified sufficient restrictions to guarantee that a bound of this form holds. Their restrictions are again related to Nagai's conjecture; see [9] and Green, et al. [6] for details.

\section{References}

[1] A. Beauville, Variétés Kähleriennes dont la première classe de Chern est nulle, J. Differential Geom. 18 (1983), no. 4, 755-782.

[2] A. Beauville, private communication, 1999.

[3] A. Fujiki, On primitively symplectic compact Kähler V-manifolds of dimension four, in Classification of algebraic and analytic manifolds (Katata, 1982), 71-250, Progr. Math. 39, Birkhäuser Boston, Boston, MA, 1983. 
[4] W. Fulton and J. Harris, Representation theory: a first course, Graduate texts in mathematics 129, Springer-Verlag, New York, 1991.

[5] L. Göttsche and W. Soergel, Perverse sheaves and the cohomology of Hilbert schemes of smooth algebraic surfaces, Math. Ann. 296 (1993), no. 2, 235-245.

[6] M. Green, Y.-J. Kim, R. Laza, and C. Robles, The LLV decomposition of hyper-Kähler cohomology, preprint arXiv:1906.03432.

[7] D. Guan, On the Betti numbers of irreducible compact hyperkähler manifolds of complex dimension four, Math. Res. Lett. 8 (2001), no. 5-6, 663-669.

[8] D. Huybrechts, Finiteness results for compact hyperkähler manifolds, J. Reine Angew. Math. 558 (2003), 15-22.

[9] Y.-J. Kim and R. Laza, A conjectural bound on the second Betti number for hyper-Kähler manifolds, Bull. Soc. Math. France 148 (2020), no. 3, 467-480.

[10] N. Kurnosov, Boundness of $b_{2}$ for hyperkähler manifolds with vanishing odd-Betti numbers, preprint arXiv:1511.02838.

[11] E. Looijenga and V. Lunts, A Lie algebra attached to a projective variety, Invent. Math. 129 (1997), no. 2, 361-412.

[12] G. Mongardi, A. Rapagnetta, and G. Saccà, The Hodge diamond of O'Grady's six-dimensional example, Compos. Math. 154 (2018), no. 5, 984-1013.

[13] Y. Nagai, On monodromies of a degeneration of irreducible symplectic Kähler manifolds, Math. Z. 258 (2008), no. 2, 407-426.

[14] K. O'Grady, A new six-dimensional irreducible symplectic variety, J. Algebraic Geom. 12 (2003), no. $3,435-505$.

[15] S. Salamon, On the cohomology of Kähler and hyper-Kähler manifolds, Topology 35 (1996), no. $1,137-155$.

[16] M. Verbitsky, Actions of the Lie algebra of $\mathrm{SO}(5)$ on the cohomology of a hyper-Kähler manifold, (English translation) Funct. Anal. Appl. 24 (1990), no. 3, 229-230.

[17] M. Verbitsky, Cohomology of compact hyper-Kähler manifolds and its applications, Geom. Funct. Anal. 6 (1996), no. 4, 601-611.

Department of Mathematics

sawon@email.unc.edu

University of North Carolina

www.unc.edu/ sawon

Chapel Hill NC 27599-3250

USA 